\title{
CONNECTED COMPONENTS OF MODULI SPACES
}

\author{
F. CATANESE
}

\section{Introduction}

Let $S$ be a minimal surface of general type (complete and smooth over $\mathbb{C}$ ), and let $\mathscr{M}=\mathscr{M}(S)$ (resp., $\mathscr{M}^{\text {diff }}$ ) be the coarse moduli space of complex structures on the oriented topological (resp., differential) 4-manifold underlying $S$.

By Gieseker's theorem [5], $\mathscr{M}(S)$ is a quasiprojective variety, and the number $\nu(S)$ of its irreducible components is bounded by a function $\nu_{0}\left(K^{2}, \chi\right)$ of the two (topological) invariants $K^{2}=K_{S}^{2}, \chi=\chi\left(\mathcal{O}_{S}\right)$.

Let $\lambda(S)$ be the number of connected components of $\mathscr{M}(S)$ : this short note answers a question raised in a previous paper [1], showing that the above number $\lambda(S)$ can be arbitrarily large.

As in [1], to which we shall constantly refer, again we restrict our attention to bidouble (i.e., Galois with group $\left.(\mathbb{Z} / 2)^{2}\right)$ covers of $Q=\mathbb{P}^{1} \times \mathbb{P}^{1}$ : indeed, (cf. [2]) we conjecture a stronger result to hold true, namely that many of the different irreducible components of $\mathscr{M}$ we thus obtain are in fact connected components of $\mathscr{M}$.

The idea of proof is rather simple: if $S$ and $S^{\prime}$ are deformations of each other, then there exists a diffeomorphism $f: S \rightarrow S^{\prime}$ such that $f^{*}\left(K_{S^{\prime}}\right)=K_{S}$ $\in H^{2}(S, \mathbb{Z})$, and, in particular, if $r(S)=\max \left\{r \in \mathbb{N} \mid(1 / r) K_{S} \in H^{2}(S, \mathbb{Z})\right\}$, then $r(S)=r\left(S^{\prime}\right)$.

In view of Donaldson's recent result [3], it is possible that the integer $r(S)$ could be an invariant of the differentiable structure for these surfaces; it is not clear at the moment whether nicer properties are enjoyed by the moduli spaces $\mathscr{M}^{\text {diff }}(S)$. Nevertheless, when the complex dimension is at least 3 , it seems (cf. [6], [7]) that similar phenomena of high disconnectedness should appear also for $\mathscr{M}^{\text {diff }}$.

Received December 27, 1985. Research partly supported by Ministero Pubblica Istruzione. 


\section{Statement and proof of the main result}

Theorem. For each natural number $k$ there exist minimal models $S_{1}, \cdots, S_{k}$ of surfaces of general type such that

(a) $S_{i}$ is simply-connected $(i=1, \cdots, k)$,

(b) for $i \neq j, S_{i}$ and $S_{j}$ are (orientedly) homeomorphic but not a deformation of each other.

Remark 1. From the proof it shall also follow that the number of moduli of $S_{i}$ (cf. [1, p. 484]) differs from the number of moduli of $S_{j}$ for $i \neq j$.

Let us recall an arithmetical result, proved by E. Bombieri in the Appendix to [1].

Lemma 2. For each positive integer $k$, there exist integers $m, T$, and $k$ distinct factorizations of $6^{m}$,

$$
u_{i}^{\prime} v_{i}^{\prime}=6^{m} \quad(i=1, \cdots, k),
$$

together with integers $w_{i}, z_{i}(i=1, \cdots, k)$ such that, setting $u_{i}=T u_{i}^{\prime}$ and $v_{i}=T v_{i}^{\prime}$, the following system of equalities and inequalities is satisfied for $i=1, \cdots, k$ :

$$
\begin{aligned}
& u_{i} v_{i}=T 6^{m}=M, \quad w_{i} z_{i}-2\left(u_{i}+v_{i}\right)=N \\
& \left(u_{i}+2\right) / 3<w_{i}<u_{i}-4, \quad\left(v_{i}+2\right) / 3<z_{i}<v_{i}-4 .
\end{aligned}
$$

Corollary 3. In the notations of Lemma 2, the greatest common divisors $\left(u_{i}, v_{i}\right)$ assume at least $k / 2$ distinct values.

Proof. Set $u_{i}^{\prime}=2^{x_{i}} 3^{y_{i}}$. We can clearly assume $x_{i} \leqslant m-x_{i}$, hence $\left(u_{i}, v_{i}\right)$ $=T 2^{x_{i}} 3^{\min \left(y_{i}, m-y_{i}\right)}$. Since the factorizations are distinct, $\left(u_{i}, v_{i}\right)=\left(u_{j}, v_{j}\right)$ for $i \neq j$ if and only if $x_{i}=x_{j}, y_{i}=m-y_{j}$. q.e.d.

Given a smooth projective variety $X$ we denote by $\mathrm{NS}(X)$ the Neron-Severi group of divisors modulo numerical equivalence (which we shall denote by $\sim$, leaving the symbol $\equiv$ for linear equivalence). Note that, more generally on a compact complex manifold $X$,

$$
\operatorname{NS}(X)=\left(\operatorname{ker}\left(H^{2}(X, \mathbb{Z}) \rightarrow H^{2}\left(X, \mathcal{O}_{X}\right)\right)\right) / \text { torsion. }
$$

Lemma 4. Let $X, Y$ be smooth projective varieties and let $\pi: X \rightarrow Y$ be $a$ finite Galois cover with group $G$. Then

(i) $\pi^{*}: \mathrm{NS}(Y) \rightarrow \mathrm{NS}(X)$ is injective, maps to $L=\left(\operatorname{ker} \pi_{*}\right)^{\perp}=\mathrm{NS}(X)^{G}$, and $L / \mathrm{im} \pi^{*}$ is a torsion subgroup of exponent at most the order of $G$.

If $B_{1}, \cdots, B_{k}$ are the irreducible components of the branch divisor $B$ of $\pi$, let ( for $i=1, \cdots, k) e_{i}$ be the order of the inertia group of any divisor in $\pi^{-1}\left(B_{i}\right)$, let $d_{i}$ be the order of divisibility of the class of $B_{i}$ in $\operatorname{NS}(Y)\left(d_{i}=\right.$ $\max \left\{d \mid \exists \Gamma_{i}\right.$ s.t. $\left.\left.d \Gamma_{i} \sim B_{i}\right\}\right)$, and set $m_{i}=$ g.c.d. $\left(e_{i}, d_{i}\right), a_{i}=e_{i} / m_{i}$. 
Assume furthermore $H_{1}(X, \mathbb{Z})=0$ and $H^{2}\left(G, \mathbb{C}^{*}\right)=0$ (e.g., if $G$ is cyclic). Then

(ii) the exponent $\beta$ of $L / \operatorname{Im} \pi^{*}$ is the least common multiple $\alpha$ of the numbers $a_{1}, \cdots, a_{k}$.

Proof. If $m$ is the order of the group $G$, we have $\pi_{*} \pi^{*}=m$ (Identity), hence $\pi^{*}$ is injective, and $\operatorname{im} \pi^{*} \subset\left(\operatorname{ker} \pi_{*}\right)^{\perp}$ by the projection formula $\pi^{*} x \cdot y=x \cdot \pi_{*} y$. Moreover, since $\pi^{*} \pi_{*}=\sum_{g \in G} g^{*}$, tensoring over $\mathbb{Q}$, $\operatorname{ker} \pi_{*}$ is the kernel of the projector onto the subspace of invariants, and $\left(\operatorname{ker} \pi_{*}\right)^{\perp}=$ $\operatorname{NS}(X)^{G}$. If $x \in L$, then $g^{*} x=x \forall g \in G$; hence $m x=\pi^{*}\left(\pi_{*} x\right)$ and the first assertion is proven.

Since $H_{1}(X, \mathbb{Z})=0$, any element $x$ in $L$ is represented by a divisor $D$ s.t. $g^{*} D \equiv D \forall g \in G$.

Consider the sheaf $\mathscr{L}=\mathcal{O}_{X}(D)$ of rational functions $f$ with $\operatorname{div}(f)-D \geqslant$ 0 : by assumption, $\forall g \in G$ there exists an isomorphism between $\mathscr{L}$ and $g^{*} \mathscr{L}$, hence, defining $G(\mathscr{L})=\left\{(g, \tilde{g}) \mid g \in G\right.$ and $\tilde{g}$ is an isomorphism from $g^{*} \mathscr{L}$ to $\mathscr{L}\}$, we have a central extension

$$
0 \rightarrow \mathbb{C}^{*} \rightarrow G(\mathscr{L}) \rightarrow G \rightarrow 0 .
$$

We notice that

Sublemma 6. (5) splits if and only if $D$ is linearly equivalent to a $G$-invariant divisor $D^{\prime}$ (i.e., $g\left(D^{\prime}\right)=D^{\prime} \forall g \in G$ ).

Proof. The "if" part is obvious, since then $\mathscr{L} \cong \mathcal{O}\left(D^{\prime}\right)$ and the condition $\operatorname{div}(f)-D^{\prime} \geqslant 0$ is clearly $G$-invariant, hence there is an action of $G$ on $\mathscr{L}$ which makes (5) split. Conversely, if (5) splits there is an action of $G$ on $\mathscr{L}$, and the sheaf $\left(\pi_{*} \mathscr{L}\right)^{G}$ is nonzero.

If $H$ is a very ample divisor on $Y=X / G$, for $m \gg 0$ the sheaf $\left(\pi_{*} \mathscr{L}\right)^{G}(m H)$ has a section, hence $D+m \pi^{*} H$ is linearly equivalent to an effective divisor $C$, which is $G$-invariant. q.e.d.

Now the extensions of $G$ by $\mathbb{C}^{*}$ are classified by $H^{2}\left(G, \mathbb{C}^{*}\right)$; hence, if $H^{2}\left(G, \mathbb{C}^{*}\right)=0, D$ is linearly equivalent to a $G$-invariant divisor, and we can only consider the case of an effective $G$-invariant divisor $C$. In this case, if $R_{i}=\pi^{-1}\left(B_{i}\right)_{\text {red }}$, we can write $C$ as $C=C_{R}+C^{\prime}$, where $C_{R}, C^{\prime}$ are effective, no component of the ramification divisor $R$ appears in $C^{\prime}$, and $C_{R}=\sum_{i=1}^{k} b_{i} R_{i}$ (since $g(C)=C \forall g \in G$, this is possible).

We have

$$
\pi_{*}(C)=m \Gamma^{\prime}+\sum_{i}\left(b_{i} m / e_{i}\right) B_{i} \sim m \Gamma^{\prime}+\sum_{i}\left(b_{i} d_{i} m / e_{i}\right) \Gamma_{i}
$$

since $B_{i}$ is exactly $d_{i}$-divisible.

Now

$$
m C \equiv \pi^{*} \pi_{*}(C) \equiv m \pi^{*}\left(\Gamma^{\prime}\right)+\sum_{i}\left(b_{i} d_{i} m / e_{i}\right) \pi^{*}\left(\Gamma_{i}\right)
$$


hence

$$
\alpha C \equiv \pi^{*}\left(\alpha \Gamma^{\prime}\right)+\sum_{i}\left(\alpha / a_{i}\right) b_{i}\left(d_{i} / m_{i}\right) \pi^{*}\left(\Gamma_{i}\right),
$$

thus $\alpha C$ belongs to im $\pi^{*}$.

Conversely, we claim that the class of $R_{i}$ in $L / \mathrm{im} \pi^{*}$ has period exactly equal to $a_{i}$.

In fact $a_{i} R_{i} \equiv\left(d_{i} / m_{i}\right) \pi^{*}\left(\Gamma_{i}\right)$, as we have seen, and if there exists a divisor $\Gamma$ and some integer $c$ dividing $a_{i}$ such that $c R_{i} \equiv \pi^{*}(\Gamma)$, applying $\pi_{*}$ we get

$$
m \Gamma \sim c \pi_{*}\left(R_{i}\right) \sim\left(\mathrm{cm} / e_{i}\right) B_{i} .
$$

Hence $e_{i} \Gamma \sim c B_{i} \sim c d_{i} \Gamma_{i}$, thus $\left(e_{i}=a_{i} m_{i} !\right) \quad a_{i} m_{i} \Gamma \sim\left(c m_{i} d_{i} / m_{i}\right) \Gamma$ and $\left(d_{i} / m_{i}\right) \Gamma_{i} \sim a_{i} / c \Gamma$. Since $d_{i} / m_{i}$ and $a_{i} / c$ are relatively prime, $\Gamma_{i}$ is $a_{i} / c$ divisible, therefore $a_{i}=c$.

Remark. The above proof shows that, in general, $\beta \geqslant \alpha$.

Corollary 7. Let $\pi: X \rightarrow Y$ be a finite Galois cover with group $G$ s.t. $\pi$ is the composition of Galois covers as in (ii) of Lemma 4, each such that the corresponding integer $\alpha$ equals 1 . Then $\mathrm{NS}(X)^{G}=\pi^{*}(\mathrm{NS}(Y))$.

Proof. The proof is by induction on the number of steps: in fact if $N$ is a normal subgroup of $G$ and $Z=X / N$ is smooth, let $p: X \rightarrow Z, q: Z \rightarrow Y$ be the quotient morphisms. Since $p^{*}$ and $q^{*}$ are injective by Lemma 4, we can identify $\mathrm{NS}(Y)$ and $\mathrm{NS}(Z)$ to subgroups of the free abelian group $\mathrm{NS}(X)$.

Let $\Gamma=G / N$ : by induction $\mathrm{NS}(Y)=\mathrm{NS}(Z)^{\Gamma}=\left(\mathrm{NS}(X)^{N}\right)^{\Gamma}=\mathrm{NS}(X)^{G}$. q.e.d.

Remark. The result of Corollary 7 can be stated in a greater generality, in particular one does not need the intermediate quotients to be smooth.

Proof of the theorem. Recall that $\pi: S \rightarrow Q=\mathbb{P}^{1} \times \mathbb{P}^{1}$ is a smooth simple bidouble cover of type $(2 a, 2 b),(2 n, 2 m)$ if $\pi$ is a finite $(\mathbb{Z} / 2)^{2}$ Galois cover, $S$ is a smooth surface, and the branch locus of $\pi$ consists of two curves of respective bidegrees $(2 a, 2 b),(2 n, 2 m)$.

Apply Lemma 2 to the integer $2 k$. Then, for $i=1, \cdots, 2 k$ set (in the notations of the lemma)

$$
\begin{aligned}
& a_{i}=\left(u_{i}+w_{i}\right) / 2+1, \quad n_{i}=\left(u_{i}-w_{i}\right) / 2+1, \\
& b_{i}=\left(v_{i}-z_{i}\right) / 2+1, \quad m_{i}=\left(v_{i}+z_{i}\right) / 2+1,
\end{aligned}
$$

and let, for $i=1, \cdots, 2 k, \pi_{i}: S_{i} \rightarrow Q$ be a smooth simple bidouble cover of type $\left(2 a_{i}, 2 b_{i}\right),\left(2 n_{i}, 2 m_{i}\right)$. As in [1], p. 506] we see that

$$
K_{S_{i}}^{2}=8 M, \quad \chi\left(\mathcal{O}_{S_{i}}\right)=\frac{3}{2} u_{i} v_{i}+\left(u_{i}+v_{i}\right)+2-\frac{1}{2} w_{i} z_{i}=\frac{3}{2} M+2-\frac{1}{2} N .
$$

Moreover,

$$
K_{S_{i}}=\pi_{i}^{*}\left(\mathcal{O}_{Q}\left(u_{i}, v_{i}\right)\right)
$$


and, $u_{i}, v_{i}$ being even, $K_{S_{i}}$ is 2-divisible: hence, by Freedman's theorem [4] (cf. also [1, Theorem 4.6]), all the surfaces $S_{i}$ are (orientedly) homeomorphic. Applying Corollary 6 to $\pi_{i}: S_{i} \rightarrow Q$, and using (8), we see that $r\left(S_{i}\right)=$ $\max \left\{r \in \mathbb{N} \mid(1 / r) K_{S_{i}} \in H^{2}\left(S_{i}, \mathbb{Z}\right)\right\}$ equals the greatest common divisor $\left(u_{i}, v_{i}\right)$.

By Corollary 3 there are at least $k$ of the $2 k$ surfaces $S_{1}, \cdots, S_{2 k}$, which satisfy the requirements of the theorem ( $S_{i}$ is simply connected by [1, Proposition 2.7]), since $r(S)$ is a deformation invariant, as is easy to show.

\section{References}

[1] F. Catanese, On the moduli spaces of surfaces of general type, J. Differential Geometry 19 (1984) 483-515.

[2] _ Automorphisms of rational double points and moduli spaces of surfaces of general type, Compositio Math., to appear.

[3] S. Donaldson, La topologie differentielle des surfaces complexes, C. R. Acad. Sci. Paris Sér. I. Math. 301 (1985) 317-320.

[4] M. H. Freedman, The topology of four dimensional manifolds, J. Differential Geometry 17 (1982) 357-453.

[5] D. Gieseker, Global moduli for surfaces of general type, Invent. Math. 43 (1977) 233-282.

[6] A. Libgober \& J. Wood. Differentiable structures on complete intersections. I, Topology 214 (1982) 469-482.

[7] _ Differentiable structures on complete intersections. II, Proc. Sympos. Pure Math., Vol. 40, Part 2, Amer. Math. Soc., Providence, RI, 1983, 123-133.

[8] Y. Namikawa, Periods of Enriques surfaces, Math. Ann. 270 (1985) 201-222.

Universita di Pisa, ITALY 
